\title{
Fast Synthesis of Ligand-Coordinated Chromium (II) Acetate with Quadruple Bonds by Seed-Mediated Growth
}

\author{
Received 1 June, 2021; revised 21 July, 2021; accepted 29 July, 2021
}

\author{
Intek Song* \\ Department of Applied Chemistry, Andong National University, Andong 36729, Republic of Korea
}

*Corresponding author E-mail: songintek@anu.ac.kr

\begin{abstract}
A new way to prepare single crystals of ligand-coordinated chromium (II) acetate with quadruple bonds is presented. Coordination of water and pyridine with crystal growth occur by seed-mediated growth. The product was obtained as crystals with well-defined facets. Raman spectroscopy confirmed the high quality and purity of the products. Mechanism studies showed that the growth reaction is driven by heterogeneous crystallization at the interface of the seeds. Seed-mediated growth results in the high quality and large size of the crystals with improved yield for shorter reaction time.
\end{abstract}

Keywords: Chromium, Quadruple bonds, Seed-mediated growth, Crystallization, Solid-liquid interface

\section{Introduction}

Many coordination complexes have unique quadruple bonds between the metal centers [1]. The electronic configuration of the quadruple bonds is $\sigma^{2} \pi^{4} \delta^{2}$. The $\delta$ orbitals are formed by the overlap of the $\mathrm{d}$ orbitals that are in eclipsed positions. Paddlewheel ligands are often employed to interlock the metal centers so that the $d$ orbitals stay eclipsed. These paddlewheel structures can leave an empty site along the axis of the quadruple bonds where additional ligands can be coordinated $[1,2]$ [Fig. 1(a)]. And the basicity of the "axial" ligands affects the intermetallic distance [1-3]. When strongly basic ligands are coordinated, they donate more electrons to the metal centers, so the intermetallic bonds are weakened. The weakened bonds consequently elongate the intermetallic distance. Then, the longer the distance of the intermetallic bonds, the narrower the energy gap between the highest occupied molecular orbital (HOMO) and the lowest unoccupied molecular orbital (LUMO). Indeed, Cotton et al. [3] reported that controlling the energy gap between HOMO and LUMO is feasible with proper axial ligands. And this allows transition metal complexes with quadruple bonds to be promising candidates for high-performance semiconductor materials with tunable band gap. The transition between the singlet state of the ground state and the triplet state of the excited state also suggests that narrowing the energy gap also facilitates its applications for controllable magnetism.

Among various complexes with quadruple bonds, chromium (II) acetate $\left[\mathrm{Cr}_{2}(\mathrm{OAc})_{4}\right]$ is a representative example that can resist oxidation in the air for a couple of hours $[1,4,5]$ [Fig. 1(a)]. And there are numerous ligands that can be coordinated at the axial position of the complexes. The correlation between the basicity of the ligands and the chromium acetates is well studied in previous reports $[1,2,6,7]$. Therefore, chromium (II) acetates are eligible for various applications such as electronics in that they can be handled in air and their properties can be controlled by choosing proper ligands.

Ligand-coordinated chromium (II) acetates are prepared by two- (a)

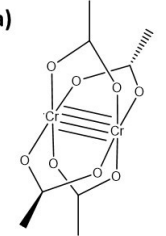

Figure 1. (a) Molecular structure of chromium (II) acetate with quadruple bonds. (b) Scheme of the seeded growth of ligand-coordinated chromium (II) acetate.

step reactions: synthesis of anhydrous chromium (II) acetate and coordination of the ligands to the anhydrous complex. In the case of the former, Levy et al. [8] has reported a new way that can be done in open air condition. And the latter is achieved by dissolving the anhydrous complex into a target solvent [2], or by exposing the vapor of the ligand into the anhydrous complex [9]. Both methods have pros and cons. The former yields high-purity products with the size of several hundreds of micrometers, but the reaction yield was low $(\sim 10 \%)$ and requires longer reaction time. The latter exhibits very high yield, but the size of the complexes is not as large as the former, and facets are not clear. Therefore, a new way that prepares ligand-coordinated chromium acetates with higher crystallinity, faster growth, and higher yield is still required.

Herein, an improved way to synthesize ligand-coordinated chromium (II) acetate is presented. Unlike the previous methods, this new method features seed-mediated growth and rapid cooling of the solution during the crystallization in a freezer [Fig. 1(b)]. This process promotes the crystallization and provides protection against oxidation. The properties of the products were then analyzed by Raman spectroscopy, which clearly tells the quality and purity of the samples. Also, the crystal growth mechanism at the solid-liquid interface of the seed and the solution was investigated based on the observations. 


\section{Experimental details}

Anhydrous chromium (II) acetate was synthesized by the method after Levy et al. [8]. Note that $\mathrm{HCl}$ was used instead of $\mathrm{HBr}$, and the amount of chromium powder was reduced by half. The total reaction time was $1.5 \mathrm{~h}$. Halving the amount of reactant without reducing the reaction time results in the minimization of the amount of by-product or residual reactant. Powdery, red specimen was obtained after the precipitation and the filtration [Fig. 2(a)]. Note that acetone was used to rinse the product.

The ligands were then coordinated by dissolving the anhydrous chromium (II) acetate powder into the target ligand solvents. Before the dissolution, the ligand solvents $(120 \mathrm{ml})$ were degassed by heating the solvent near the boiling temperature with supplying Ar gas. After $30 \mathrm{~min}, 1 \mathrm{~g}$ of the anhydrous powder was put into the degassed solution while being stirred. When the dissolution was completed, the stirring process was immediately stopped and taken out of the heating bath. The solution was then rapidly cooled down to $-20^{\circ} \mathrm{C}$ in a freezer under inert gas condition; a balloon of Ar gas was attached to the vessel to prevent the infusion of oxygen in the air. After $3 \mathrm{~h}$, red crystals precipitated at the bottom were retrieved by thawing and filtering the frozen solution at room temperature $\left(20^{\circ} \mathrm{C}\right)$.

For Raman spectroscopy, Alpha 300R (WITec) was used with excitation laser of Nd:YAG $(532.2 \mathrm{~nm}, 0.5 \mathrm{~mW})$. All measurements were carried out in air at room temperature $\left(20^{\circ} \mathrm{C}\right)$.

\section{Results and discussion}

Anhydrous chromium (II) acetate was received as red powder [Figs. 2(a) and 2(d)]. Water-coordinated chromium (II) acetate [chromium (II) acetate hydrate] was prepared by dissolving anhydrous chromium (II) acetate into degassed water. Anhydrous chromium (II) acetate can be effectively converted to ligand-coordinated complexes by both liquid- and vapor-phase ligand, unless it is oxidized during the ligand $[2,9]$. After cooled down to $-20^{\circ} \mathrm{C}$, red crystals were found at the bottom of the flask. Note that the aqueous solution, once frozen, provides protection against oxidation [Fig. 2(b)]. The diameter of crystal is submillimeter $(\sim 0.5 \mathrm{~mm})$, which is comparable or slightly larger to the direct dissolution by Song et al. [2], and an order of the magnitude larger than ligand vapor exposure method by Park et al. [9] [Fig. $2(\mathrm{e})]$. However, the yield by the current method was $30.16 \%$, which is roughly three times higher than Song et al. [2] ( 10\%). Raman spectroscopy also confirmed that the product is ligand-coordinated chromium (II) acetate without any sign of other byproducts [2] (Fig 3). The red shift of the peak near $700 \mathrm{~cm}^{-1}$ in the water-coordinated complex corresponds to the coordination of the water at the axial position, which donates electron lone pairs that weaken the intermetallic quadruple bond. This shows that this new method improves the
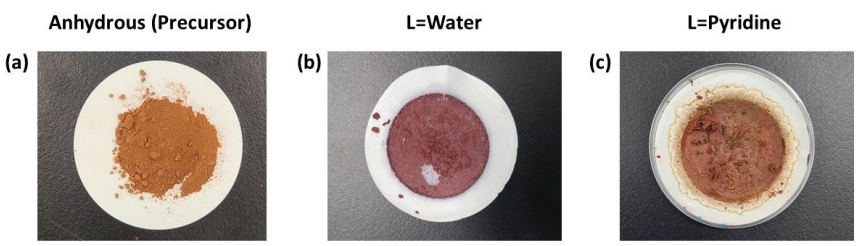

(d)

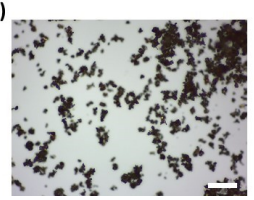

(e)

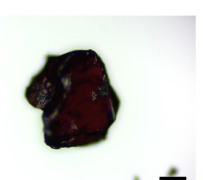

(f)

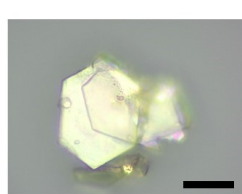

Figure 2. (a) to (c): Photograph of anhydrous (a), water-coordinated (b), and pyridine-coordinated (c) chromium (II) acetate [where $\mathrm{L}$ denotes $\mathrm{Cr}_{2}(\mathrm{OAc})_{4} \mathrm{~L}_{2}$ ]. (d) to (f): Optical microscopy images of crystals from (a) to (c), respectively. The scale bar denotes $100 \mu \mathrm{m}$ for (d) and (e) and $25 \mu \mathrm{m}$ for (f)
Wavelength $(\mathrm{nm})$ $\begin{array}{lllllllll}540 & 544 & 548 & 552 & 556 & 560 & 564 & 568 & 572\end{array}$

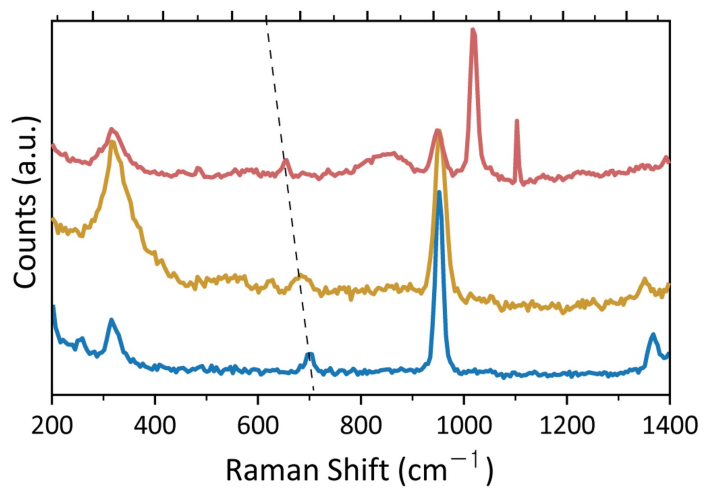

Figure 3. Raman spectra of the anhydrous (blue), water- (yellow) and pyridinecoordinated (red) specimens. The dashed line shows the shift of the peaks depending on the basicity of the axial ligands.

yield without changing the quality and purity of the product for shorter time.

In addition, pyridine can be used for the reaction [Fig. 2(c)]. Both water and pyridine have lone pairs that can form coordination bonding with chromium (II) acetate. However, the cooling temperature, $-20^{\circ} \mathrm{C}$, is not enough to freeze pyridine, because its melting point is $-42^{\circ} \mathrm{C}$. Indeed, after the cooling process, the solution was not frozen. But small red crystals were also precipitated at the bottom of the vessel. The size of the crystals reached $50 \mu \mathrm{m}$ in diameter, which also comparable to the previous report [2] [Fig. 2(f)]. Its Raman spectrum also matches with the previously reported spectrum, and no other contaminants or residues were found (Fig. 3). The yield was estimated 19.07 $\%$, which is also higher than the previous report. This shows that the current method can be applied to ligands other than water. And freezing the solvent is not necessary for the improved crystallization; the temperature gradient is the crucial factor.

When hexane was used for the reaction, small crystals were dispersed right after putting the anhydrous powder. The dispersed powder rapidly sank down at the bottom of the vial. And no coordination product was obtained. These results match with previous reports. $[1,2,9]$ Hexane is not capable of forming coordination bonds with chromium (II) acetate, because it does not have any lone pairs to donate. Hexane therefore does not dissolve chromium (II) acetate. This shows that crystallites can be dispersed right after putting the anhydrous powder regardless of the dissolution or coordination.

One of the major differences of this new method from the previous methods is the rapid cooling of the "unclear" solution. In general, rapid cooling results in the small size of the crystals. The retention of the crystal size and the higher yield, despite faster reaction, imply that the reaction mechanism is different from the previous methods. The opaqueness of the solution right before the crystallization implies that there are particulates floating in the solution, forming a (quasi-) colloidal suspension. To assure the presence of the small particulates in the solution, a small portion of the solution was drop-casted onto a substrate. And optical microscopy revealed the presence of small crystals in the solution (Fig. 4). The size of the crystallites was approximately $10 \mu \mathrm{m}$, which is considerably smaller than the size of the final product. Since chromium (II) acetate can undergo ligand coordination by simple exposure of the ligand vapor, such dispersion in solvent can facilitate the ligand coordination [9]. This implies that these small crystallites serve as nucleation sites during the growth reaction. The previous report shows that the growth without the seeds, i.e., using clear supernatant only, requires longer time (up to $24 \mathrm{~h}$ ) to achieve the comparable size of the crystals [2].

Therefore, the presence of tiny crystallites before the cooling process is the key to the improvement. In general, crystallization is com- 


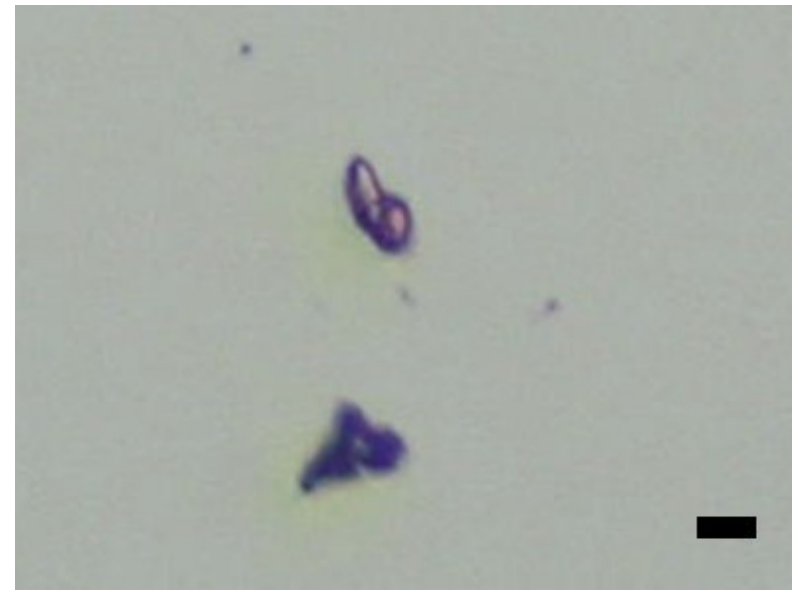

Figure 4. Optical microscopic image of the crystallites formed at the beginning of the dissolution process. The scale bar denotes $10 \mu \mathrm{m}$.

posed of two steps: nucleation and growth [10]. The nucleation step involves the generation of nucleation seeds, and the growth step accompanies the increase in the size of pre-existing seeds. In pristine solution, the nucleation occurs by the self-agglomeration of the solutes in supersaturated solution, but this process is kinetically slow. In the previous method by Song et al. [2], only the clear supernatant was used for crystallization. Therefore, the nucleation should be preceded during the reaction. In contrast, the current method uses unclear solution that already contains the crystallites. And these crystallites become seeds during the growth of crystals, skipping the nucleation step. Such provision of nucleation seeds boosts the crystallization, and the resulting crystals are very large and shows high quality, as shown in other solution-based synthesis process [11]. The large temperature gradient formed by the rapid cooling process then accelerates the growth of the crystals at the interface of the solid seed and the solution liquid. That is, heterogeneous crystallization occurs. In heterogeneous crystallization, crystallization preferentially occurs at the interface of the solid-liquid interface. It lowers the energy barrier that hinders the formation of the growing nucleus. And in the current method the interfaces between the small crystallites (solid) and the solution (liquid) allows heterogeneous nucleation.

\section{Conclusions}

In conclusion, the heterogeneous crystallization of ligand-coordinated chromium (II) acetate is achieved by rapid cooling of the solution. When compared with the products obtained by Song et al. [2], the current method features increased yield and faster reaction while retaining the size of the product. The crystals is larger and more well-defined than vapor-based method by Park et al. [9]. It shows that the coordination and the crystallization is much faster and efficient than both of the previous reports. The main driving force for such improvement is the presence of small crystallites formed at the beginning of dissolution process. And this allows facile crystal growth at the interface of the nucleus and the solution that lowers the energy barrier. The new findings in this report would contribute to the development of coordination reaction with improved efficiency.

\section{Acknowledgements}

This work was supported by a grant from 2019 Research Fund of Andong National University.

\section{References}

[1] F. A. Cotton, C. A. Murillo, and R. A. Walton, Multiple Bonds Between Metal Atoms (Springer-Verlag, New York, 2005).

[2] I. Song, J. Koo, and S. M. Yoon, RSC Adv. 9, 24319 (2019).

[3] F. A. Cotton, H. Chen, L. M. Daniels, and X. Feng, J. Am. Chem. Soc. 114, 8980 (1992).

[4] F. A. Cotton, B. G. DeBoer, M. D. Laprade, J. R. Pipal, and D. A. Ucko, J. Am. Chem. Soc. 92, 2926 (1970).

[5] F. A. Cotton, B. G. DeBoer, M. D. LaPrade, J. R. Pipal, and D. A. Ucko, Acta Cryst. B 27, 1664 (1971).

[6] F. A. Cotton and G. W. Rice, Inorg. Chem. 17, 2004 (1978).

[7] F. A. Cotton, M. Extine, and G. W. Rice, Inorg. Chem. 17, 176 (1978).

[8] O. Levy, B. Bogoslavsky, and A. Bino, Inorganica Chim. Acta 391, 179 (2012).

[9] J. Park and I. Song, Appl. Sci. Converg. Technol. 29, 99 (2020).

[10] R. P. Sear, CrystEngComm 16, 6506 (2014).

[11] M. Pradhan, I. Song, J. Lee, M. Lee, C. Park, and H. C. Choi, RSC Adv. 6, 106960 (2016). 